Linköping studies in science and technology

Licentiate thesis No. 1790

\title{
Study of novel electronic materials by mid- infrared and terahertz optical Hall effect
}

Nerijus Armakavicius

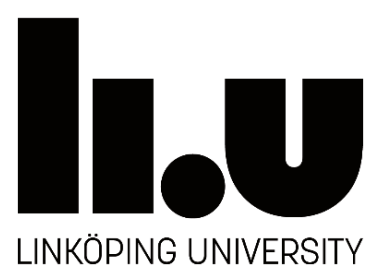

Department of Physics, Chemistry and Biology (IFM)

Linköping University, SE-581-83, Sweden

Linköping 2017 
During the course of research underlying this thesis, Nerijus Armakavicius was enrolled in Agora Materiae, a multidisciplinary doctoral program at Linköping University, Sweden.

(C) Nerijus Armakavicius, 2017

Published article has been reprinted with the permission of the copyright holder.

Printed in Sweden by LiU-Tryck, Linköping, Sweden, 2017

ISBN 978-91-7685-433-4

ISSN 0280-7971 


\begin{abstract}
Development of silicon based electronics have revolutionized our every day life during the last three decades. Nowadays Si based devices operate close to their theoretical limits that is becoming a bottleneck for further progress. In particular, for the growing field of high frequency and high power electronics, Si cannot offer the required properties. Development of materials capable of providing high current densities, carrier mobilities and high breakdown fields is crucial for a progress in state of the art electronics.

Epitaxial graphene grown on semi-insulating silicon carbide substrates has a high potential to be integrated in the current planar device technologies. High electron mobilities and sheet carrier densities make graphene extremely attractive for high frequency analog applications. One of the remaining challenges is the interaction of epitaxial graphene with the substrate. Typically, much lower free charge carrier mobilities, compared to free standing graphene, and doping, due to charge transfer from the substrate, is reported. Thus, a good understanding of the intrinsic free charge carriers properties and the factors affecting them is very important for further development of epitaxial graphene.

III-group nitrides have been extensively studied and already have proven their high efficiency as light sources for short wavelengths. High carrier mobilities and breakdown electric fields were demonstrated for III-group nitrides, making them attractive for high frequency and high power applications. Currently, In-rich InGaN alloys and AlGaN/GaN high electron mobility structures are of high interest for the research community due to open fundamental questions.

Electrical characterization techniques, commonly used for the determination of free charge carrier properties, require good ohmic and Schottky contacts, which in certain cases can be difficult to achieve. Access to electrical properties of buried conductive channels in multilayered structures requires modification of samples and good knowledge of the electrical properties of all electrical contact within the structure. Moreover, the use of electrical contacts to electrically characterize two-dimensional electronic materials, such as graphene, can alter their intrinsic properties. Furthermore, the determination of effective mass parameters commonly employs cyclotron resonance and Shubnikov-de Haas oscillations measurements, which require long scattering times of free charge carriers, high magnetic fields and low temperatures.

The optical Hall effect is an external magnetic field induced optical anisotropy in conductive layers due to the motion of the free charge carriers under the influence of the Lorentz force, and is equivalent to the electrical Hall effect at optical frequencies. The optical Hall effect can be measured by generalized ellipsometry and provides a powerful method for the determination of free charge carrier properties in a non-destructive and contactless manner. In principle, a single optical Hall effect measurement can provide
\end{abstract}


quantitative information about free charge carrier types, concentrations, mobilities and effective mass parameters at temperatures ranging from few kelvins to room temperature and above. Further, it was demonstrated that for transparent samples, a backside cavity can be employed to enhance the optical Hall effect.

Measurement of the optical Hall effect by generalized ellipsometry is an indirect technique requiring subsequent data analysis. Parameterized optical models are fitted to match experimentally measured ellipsometric data by varying physically significant parameters. Analysis of the optical response of samples, containing free charge carriers, employing optical models based on the classical Drude model, which is augmented with an external magnetic field contribution, provide access to the free charge carrier properties.

The main research results of the graduate studies presented in this licentiate thesis are summarized in the five scientific papers.

Paper I. Description of the custom-built terahertz frequency-domain spectroscopic ellipsometer at Linköping University. The terahertz ellipsometer capabilities are demonstrated by an accurate determination of the isotropic and anisotropic refractive indices of silicon and $m$-plane sapphire, respectively. Further, terahertz optical Hall effect measurements of an $\mathrm{AlGaN} / \mathrm{GaN}$ high electron mobility structures were employed to extract the two-dimensional electron gas sheet density, mobility and effective mass parameters. Last, in-situ optical Hall effect measurement on epitaxial graphene in a gas cell with controllable environment, were used to study the effects of environmental doping on the mobility and carrier concentration.

Paper II. Presents terahertz cavity-enhanced optical Hall measurements of the monolayer and multilayer epitaxial graphene on semi-insulating $4 \mathrm{H}-\mathrm{SiC}(0001)$ substrates. The data analysis revealed p-type doping for monolayer graphene with a carrier density in the low $10^{12} \mathrm{~cm}^{-2}$ range and a carrier mobility of $1550 \mathrm{~cm}^{2} / \mathrm{V} \cdot \mathrm{s}$. For the multilayer epitaxial graphene, n-type doping with a carrier density in the low $10^{13} \mathrm{~cm}^{-2}$ range, a mobility of $470 \mathrm{~cm}^{2} / \mathrm{V} \cdot \mathrm{s}$ and an effective mass of $(0.14 \pm 0.03) m_{0}$ were extracted. The measurements demonstrate that cavity-enhanced optical Hall effect measurements can be applied to study electronic properties of two-dimensional materials.

Paper III. Terahertz cavity-enhanced optical Hall effect measurements are employed to study anisotropic transport in as-grown monolayer, quasi free-standing monolayer and quasi free-standing bilayer epitaxial graphene on semi-insulating $4 \mathrm{H}-\mathrm{SiC}(0001)$ substrates. The data analysis revealed a strong anisotropy in the carrier mobilities of the quasi freestanding bilayer graphene. The anisotropy is demonstrated to be induced by carriers scattering at the step edges of the $\mathrm{SiC}$, by showing that the mobility is higher along the step than across them. The scattering mechanism is discussed based on the results of the 
optical Hall effect, low-energy electron microscopy, low-energy electron diffraction and Raman measurements.

Paper IV. Mid-infrared spectroscopic ellipsometry and mid-infrared optical Hall effect measurements are employed to determine the electron effective mass in an $\operatorname{In}_{0.33} \mathrm{Ga}_{0.67} \mathrm{~N}$ epitaxial layer. The data analysis reveals slightly anisotropic effective mass and carrier mobility parameters together with the optical phonon frequencies and broadenings.

Paper V. Terahertz cavity-enhanced optical Hall measurements are employed to study the free charge carrier properties in a set of $\mathrm{AlGaN} / \mathrm{GaN}$ high electron mobility structures with modified interfaces. The results show that the interface structure has a significant effect on the free charge carrier mobility and that the sample with a sharp interface between an AlGaN barrier and a GaN buffer layers exhibits a record mobility of $2332 \pm 73 \mathrm{~cm}^{2} / \mathrm{V} \cdot \mathrm{s}$. The determined effective mass parameters showed an increase compared to the GaN value, that is attributed the the penetration of the electron wavefunction into the AlGaN barrier layer. 



\section{PREFACE}

The licentiate thesis is written based on knowledge and research results accumulated during the graduate studies of Nerijus Armakavicius at the Terahertz Materials Analysis center, the Department of Physics, Chemistry and Biology in the Linköping University from October 2014 to October 2017. The licentiate thesis is based on scientific papers and contains two main parts: the first part provides a brief introduction to the research field and the second part presents the main research results summarized in five scientific papers.

The graduate studies were accomplished in a close collaboration with a research group led by prof. Mathias Schubert at the University of Nebraska-Lincoln. A financial support was provided by the Swedish Research Council (VR) under Grant No. 2013 - 5580 and 2016 - 00889, the Swedish Governmental Agency for Innovation Systems (VINNOVA) under the VINNMER international qualification program, Grant No. 2011 - 03486, the Swedish Government Strategic Research Area in Materials Science on Functional Materials at Linköping University, Faculty Grant SFO Mat LiU No. 200900971, and the Swedish Foundation for Strategic Research (SSF), under Grant No. FL12 - 0181 and RIF14 - 055. 



\section{ACKNOWLEDGEMENT}

At first I would like to express my sincere gratitude to my supervisor Prof. Vanya Darakchieva. I am very thankful for giving me an opportunity to join her research group and her trust in me over the last few years. I always felt her encouragement and support to move forward that have been very important for me. Her patience in guiding me through the graduate studies and sharing her knowledge with me was of great importance. Being a friend and an authority figure at the same time is something I always admire in Vanya. Thank you!

Next I would like to thank for my co-supervisor and friend Dr. Philipp Kühne. It have been my pleasure to work with Philipp and have a chance to learn from him. His scientific point of view in everyday life and critical thinking have had a great influence on my personal development as a scientist. His uncountable advices and extensive help have kept me moving over my graduate studies up to the point I stand today. Our long discussions have always motivated me and rise my curiosity in learning new things.

I am also very grateful to my colleagues Prof. Mathias Schubert and Dr. Vallery Stanishev for their help and support. It have been my pleasure to work with you and learn from you. Our discussions have a great value for me.

I am thankful to Dr. Jr-Tai Chen and Dr. Chih-Wei Hsu for providing me with their excellent III-group nitride samples and sharing their opinion on my results. I am looking forward to our future collaborations.

Many thanks to my friends and colleagues Dr. Chamseddine Bouhafs and Sean Knight. Your help and advices were always important for me. Also, our discussions helped to refresh my mind.

I would like to express my gratitude to Prof. Rositsa Yakimova for her help and support in my activities related with epitaxial graphene.

I am very grateful to all co-authors for their contribution and help, which was very important for completing this thesis.

I am also thankful to Prof. Per Olof Holtz for taking care of me as a PhD student and an opportunity to take part in the Agora Materiae graduate school.

Big THANKS also goes to my Lithuanian friends at the IFM. I am very grateful for your kind help, advices and nice discussions at lunch table.

I would like also thank to everyone I been in touch at LiU. All people here have been very nice to me, it was my pleasure to meet you. 
viii

Last but far from the least, my gratitude words go to my family:

Esu be galo dèkingas savo tèvams ir seneliams už viską ką esu šiandien pasiekęs. Visada jaučiau, Jūsu, dideli palaikyma ir meile, kas man visuomet buvo labai svarbu. Esu nuoširdžiai dèkingas už viska ka dèl manęs padarète, už tai, kad suteikète laisve priimti sprendimus ir padrasinote, kai to reikèjo. Niekuomet to nepamiršiu, AČIŪ! 


\section{Contents}

1 Part I 1

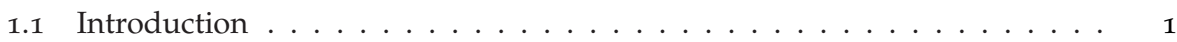

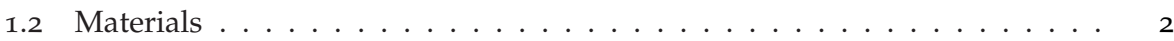

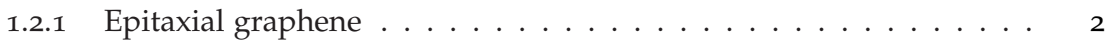

1.2.2 III-group nitrides . . . . . . . . . . . . . . 4

1.2.3 AlGaN/GaN high electron mobility transistor structures . . . . . . 5

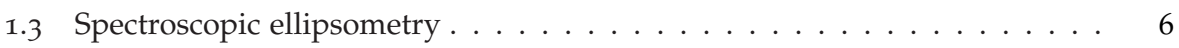

1.3.1 Standard ellipsometry . . . . . . . . . . . . . . . 6

1.3.2 Generalized ellipsometry . . . . . . . . . . . . . 8

1.3.3 Dielectric function in the infrared and terahertz spectral ranges . . . 9

1.3 .4 Optical Hall effect . . . . . . . . . . . . . . . . . 12

1.3.5 Cavity-enhanced optical Hall effect . . . . . . . . . . . . 13

1.3.6 Ellipsometric data analysis . . . . . . . . . . . . . . . 13

1.3.7 Mid-infrared spectroscopic ellipsometry and optical Hall effect mea-

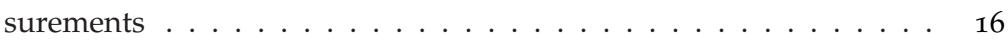

1.3.8 Terahertz optical Hall effect measurements . . . . . . . . . . . 17

$\begin{array}{lr}\text { References } & 19\end{array}$

List of abbreviations $\quad 23$

2 Part II 25

2.1 Publications included in the thesis . . . . . . . . . . . . . 25

2.2 Publications not included in the thesis . . . . . . . . . . . . 27 



\section{Part I}

\subsection{Introduction}

The development of semiconductor based electronic and optoelectronic devices have shown a tremendous progress during the last three decades that have significantly affected people's everyday life. Nowadays, silicon (Si) based electronic devices operate close to their theoretical limits in terms of the device temperatures, power densities, operational frequencies and dimensions. Due to these limitations the growth rate of Si technologies starts to level out. Moreover, the development of high power electronics has shown much slower progress and is far behind the low power electronics. To push the progress of devices operating at high frequencies and high powers, the development of new materials is crucially needed. Fundamental understanding of material properties is a key factor in materials science and requires new characterization techniques capable of providing access to the fundamental materials parameters.

Optimization of the free charge carrier (FCC) properties of materials is crucial for the improved performance of electronic devices. High FCC mobility parameters of the channel materials in transistors structures are needed to achieve the high frequency operation. Understanding of scattering mechanisms and factors affecting FCC mobility parameters is important for improving the performance of the high frequency devices. Knowledge of FCC effective mass parameter and its dependence on FCC concentration is important for understanding of material electrical and optical properties, and could help to develop modeling and designing of devices.

Contact-based electric methods, such as Hall effect and capacitance voltage measurements, are commonly used to access the FCC mobility and concentration parameters in semiconductor materials. However, they require good ohmic and Schottky contacts, which in certain cases are problematic to achieve. It is also prerequisite to have a good knowledge of the electrical properties of all contacts within sample. Electrical character- 
ization of buried conductive channels in multilayered structures requires sample modification and deposition of contacts, that might alter the intrinsic material properties. Moreover, it is problematic to assess the FCC properties in carrier type inversion and concentration graded layers.

Cyclotron resonance and Shubnikov-de Haas oscillation measurements are typically employed to determine effective mass parameters. Both methods require low temperatures and relatively high mobility parameters, and are limited to high quality materials with long scattering times. The determination of FCC properties at temperatures comparable to device operation conditions like room temperature or higher is still a challenging task.

The optical Hall effect (OHE), developed by Prof. Mathias Schubert from the University of Nebraska-Lincoln, is a new contactless method capable of overcoming the previously mentioned shortcomings for the commonly used electrical characterization techniques $[1,2]$. The $\mathrm{OHE}$ is a magnetic field induced optical birefringence in materials containing FCCs and is the equivalent of the classical Hall effect at the optical frequencies. Measurements of the OHE employ generalized ellipsometry (GE) at long wavelengths (mid-infrared (MIR) to terahertz (THz)) and can provide access to the free charge carrier properties of different conductive channels in multilayered samples in a non-destructive and contactless manner. In principle, a single measurement can provide quantitative information about FCC types, concentrations, mobilities and effective mass parameters at sample temperatures from a few kelvins to room temperature and above. The OHE is also highly sensitive to the anisotropy, capable of providing directionally resolved information of the FCC parameters.

The development of the THz ellipsometer at the Terahertz Materials Analysis center in Linköping University was part of the graduate studies research project presented in this licentiate thesis. The scope of the thesis covers the description of the $\mathrm{THz}$ ellipsometer at Linköping University and application of the MIR and THz OHE to study the FCC properties of epitaxial graphene (EG), III-group nitride epitaxial layers and AlGaN/GaN high electron mobility transistor (HEMT) structures.

\subsection{Materials}

\subsubsection{Epitaxial graphene}

The experimental discovery of graphene sparked a new research field of two-dimensional materials, which nowadays became a huge field with the graphene remaining as a core material. Graphene is atomic layer of $s p^{2}$-hybridized carbon atoms covalently bonded in a honeycomb lattice via three in-plane $\sigma$-bonds and a remaining dangling $\pi$-bond 


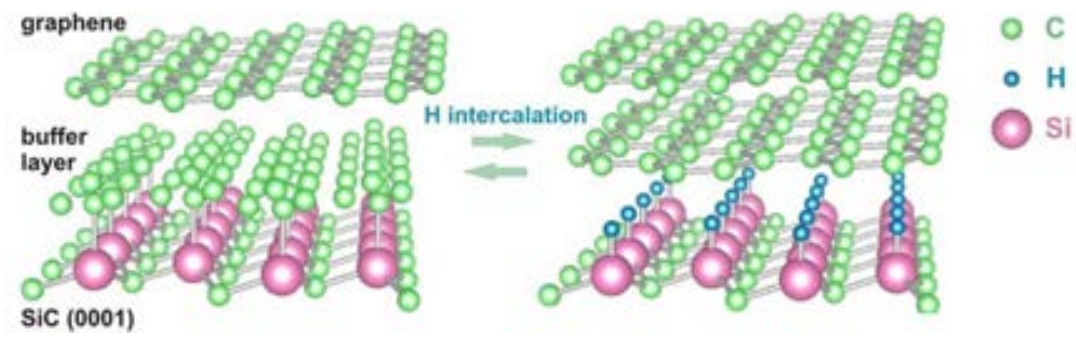

Figure 1.1: As-grown monolayer (left) and hydrogen intercalated bilayer (right) epitaxial graphene on Si-face (0001) of silicon carbide.

perpendicular to the sheet. The electronic structure of the $\pi$-band has unique linear dispersion which in two-dimensions has a form of the well-known Dirac cones formed at the $\bar{K}$ points of the first Brillouin zone. Electrons in graphene behave as massless Dirac fermions having effective speed of $\sim 10^{6} \mathrm{~m} / \mathrm{s}$ and very high FCC mobilities.

EG grown on semi-insulating silicon carbide $(\mathrm{SiC})$ substrates has a high potential for integration in the existing planar electronic devices technologies. While growth of graphene on the C-face (0001) of $\mathrm{SiC}$ substrates is difficult to control, the Si-face (0001) provides high quality, homogenous monolayer (ML) and bilayer (BL) graphene at a wafer scale, due to a self-consistent nature of the growth process [3-5]. Prototype devices based on EG have been demonstrated [6-9].

A structural scheme of the EG on Si-face (0001) is depicted in Figure 1.1 (left). Growth of $\mathrm{EG}$ on $\mathrm{SiC}$ is always accompanied with the formation of a $(6 \sqrt{3} \times 6 \sqrt{3}) R 30^{\circ}$ surface reconstructed layer of carbon atoms below, commonly called a buffer layer [3, 10]. The buffer layer does not show a Dirac-like dispersion and is electrically inactive. EG interaction with the substrate significantly affects its electronic properties. Typically, much lower FCC mobilities than for the exfoliated graphene and doping with FCCs due to interaction with the $\mathrm{SiC}$ substrate are reported for EG. Intrinsically as-grown EG is n-type doped, but exposure to the ambient environment can effect its doping significantly due to adsorption of p-type and n-type dopants [3, 11].

Hydrogen intercalation of the buffer layer reduces the interaction of EG with the substrate and produces so-called quasi-free-standing (QFS) EG. During the intercalation, the buffer layer rearranges into an additional EG layer and the remaining $\mathrm{SiC}$ surface becomes hydrogen-terminated which reduces the interaction with the EG (see Figure 1.1) [12]. Intercalation of as-grown-ML EG, having Dirac cone dispersion, produces a QFS-BL EG which is decoupled from the substrate and has a modified band structure. Typically, QFS EG shows higher FCC mobility parameter than as-grown EG, and it exhibits a p-type dop- 
ing commonly attributed to the effect of the substrate spontaneous polarization [10, 13] Hydrogen intercalation is a reversible process. It was shown that annealing of intercalated QFS EG samples in vacuum can remove the intercalation and restore the initial state of EG $[12,14]$.

\subsubsection{III-group nitrides}

Wide-bandgap semiconductors, such as III-group nitrides, $\mathrm{SiC}$ and $\mathrm{Ga}_{2} \mathrm{O}_{3}$, are the materials of choice for novel electronic and optoelectronic device applications. Their high electric breakdown fields, good thermal conductivity, high electron mobilities and saturation electron drift velocities make them highly attractive for high frequency and high power applications where conventional semiconductors, such as Si and III-group arsenides, are inferior. A great deal of efforts in the field of the wide-bandgap semiconductors is devoted to development of ( $\mathrm{Al}, \mathrm{Ga}, \mathrm{In}) \mathrm{N}$ compounds (III-group nitrides) and their ternary and quaternary alloys. Alloying III-group nitrides allows to tune their direct bandgap from the near-infrared $\left(\mathrm{InN}-E_{g}=0.65 \mathrm{eV}\right)$ to the deep-ultraviolet (AlN $-E_{g}=6.2 \mathrm{eV}$ ) completely covering the entire visible range.

$\mathrm{GaN}\left(E_{g}=3.4 \mathrm{eV}\right)$ is the best studied of all three III-group nitride binary compounds. Ga-rich InGaN based light emitting devices are nowadays widely used in blue and white solid state lightning. AlN is also a relatively well-established material often used in III-group nitride heterostructures as a nucleation layer. InN and In-rich alloys are less understood and remain active research areas. Challenges in the development of In-rich III-group nitrides mostly come from difficulties in the growth process, due to the low dissociation temperature of $\mathrm{InN}$ and high equilibrium vapor pressure of $\mathrm{N}_{2}$ that impose requirement of low growth temperatures [15]. Even though FCC mobilities reaching $3000 \mathrm{~cm}^{2} / \mathrm{V}$.s were reported and p-type doping was achieved, development of InN and In-rich InGaN alloys is still lagging behind the GaN and AlN [16, 17].

Commonly III-group nitrides are grown on foreign substrates such as sapphire and SiC. They crystallize in a hexagonal wurtzite crystal structure. The lack of an inversion symmetry of the wurtzite structure give rise to strong polarization fields along the polar $c$-axis. The main material parameters for wurtzite structure, III-group nitride binary compounds are given in Table 1.1. (Al, Ga, In)N alloys with a wurtzite structure have nine optical phonon modes which belong to the irreducible representation at the $\Gamma$ point of the Brillouin zone $[18,19]$

$$
\Gamma_{\text {opt }}=1 \mathrm{~A}_{1}+2 \mathrm{~B}_{1}+1 \mathrm{E}_{1}+2 \mathrm{E}_{2} .
$$

$\mathrm{A}_{1}$ and $\mathrm{E}_{1}$ are infrared (IR)- and Raman-active polar phonon modes which split into transverse optical (TO) and longitudinal optical $(\mathrm{LO})$ modes $\left[\mathrm{E}_{1}(\mathrm{TO}), \mathrm{E}_{1}(\mathrm{LO})\right.$ and $\mathrm{A}_{1}(\mathrm{TO}), \mathrm{A}_{1}(\mathrm{LO})$, 
Table 1.1: The main structural and optical parameters for hexagonal structure III-group nitride binary alloys

\begin{tabular}{|c|c|c|c|}
\hline & AlN & $\mathrm{GaN}$ & $\operatorname{InN}$ \\
\hline Lattice constant, a (@300 K) [nm] & 0.3112 & 0.3189 & 0.3533 \\
\hline Lattice constant, c (@300 K) [nm] & 0.4982 & 0.5185 & 0.5693 \\
\hline Relative static permittivity, $\varepsilon_{s} / \varepsilon_{0}$ & 8.5 & 8.9 & 10.5 \\
\hline Relative high frequency permittivity, $\varepsilon_{s} / \varepsilon_{0}$ & $3.8^{d}$ & $5.03^{a}$ & $6.7^{c}$ \\
\hline Bandgap (@300 K) [eV] & 6.14 & 3.43 & 0.64 \\
\hline Electron effective mass $m / m_{0}$ & $0.376^{e}$ & $0.232^{a}$ & $0.044^{b}$ \\
\hline $\mathrm{E}_{1}(\mathrm{TO})$ phonon $\left[\mathrm{cm}^{-1}\right]$ & $669^{d}$ & $560^{a}$ & $477^{c}$ \\
\hline $\mathrm{E}_{1}(\mathrm{LO})$ phonon $\left[\mathrm{cm}^{-1}\right]$ & $913^{d}$ & $742^{a}$ & 593 \\
\hline $\mathrm{A}_{1}(\mathrm{TO})$ phonon $\left[\mathrm{cm}^{-1}\right]$ & 611 & 532 & $443^{c}$ \\
\hline $\mathrm{A}_{1}(\mathrm{LO})$ phonon $\left[\mathrm{cm}^{-1}\right]$ & 890 & $732^{a}$ & 586 \\
\hline
\end{tabular}

respectively]. $\mathrm{E}_{2}^{(1)}$ and $\mathrm{E}_{2}^{(2)}$ modes are non-polar and only Raman-active, while $\mathrm{B}_{1}$ modes are IR- and Raman-inactive.

\subsubsection{AlGaN/GaN high electron mobility transistor structures}

The high potential for high frequency applications of III-group nitrides is exploited in $\mathrm{AlGaN} / \mathrm{GaN}$ HEMTs which proved to be a promising routes to achieve gigahertz $(\mathrm{GHz})$ and $\mathrm{THz}$ frequencies. GaN technology based HEMT devices operating at $200-600 \mathrm{~V}$ are already commercially available. However, GaN HEMTs still have not reached its full potential with remaining unresolved fundamental questions needed to be addressed in research community [25-28].

A high conductivity channel at the AlGaN/GaN interface, is created by the formation of a two-dimensional electron gas (2DEG) in nominally undoped materials (see Figure 1.2). The 2DEG is formed due to the differences in spontaneous and piezoelectric polarizations between $\mathrm{AlGaN}$ and $\mathrm{GaN}$ layers. Surface donor-like states in the $\mathrm{AlGaN}$ are generally believed to be source of electrons [28].

Figure 1.2 depicts a schematic of an AlGaN/GaN HEMT. SiC substrates are commonly used for GaN HEMTs due to its high thermal conductivity that improves the heat dissipation in the device. An AlN layer is typically employed as a nucleation layer for the growth of a GaN buffer layer which is followed by an AlGaN barrier layer on top. Often a thin dielectric layer is incorporated between the gate electrode and the $\mathrm{AlGaN}$ barrier (not shown in Figure 1.2) in order to reduce a gate leakage and the effect of surface traps.

The 2DEG mobility is a very important parameter for the final device performance. A prototype power amplifier based on AlGaN/GaN HEMT with a 2DEG mobility above 


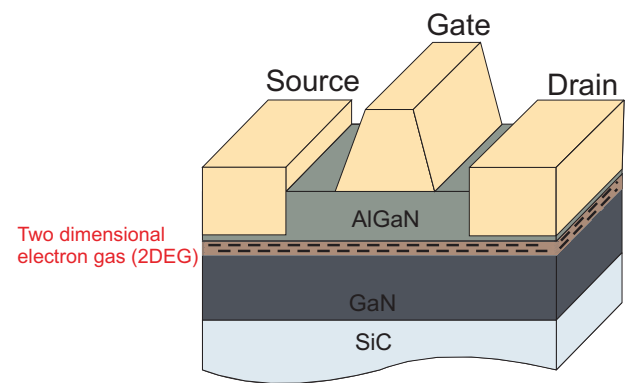

Figure 1.2: Schematic of an AlGaN/GaN high electron mobility transistor structure.

$2000 \mathrm{~cm}^{2} / \mathrm{V} \cdot \mathrm{s}$ has demonstrated a record power-gain cutoff frequency of $300 \mathrm{GHz}$ [29]. However, typically, room-temperature mobilities in the range of $1300-1600 \mathrm{~cm}^{2} / \mathrm{V} \cdot \mathrm{s}$ are reported for the AlGaN/GaN HEMTs [30, 31]. Scattering by phonons, alloy disorder and interface roughness are the main factors limiting the mobility in AlGaN/GaN HEMT structures. It was shown that the inclusion of an AlN interlayer at the AlGaN/GaN interface improves the 2DEG mobility to values above $2000 \mathrm{~cm}^{2} / \mathrm{V} \cdot \mathrm{s}$ as a result of reduced alloy disorder scattering [32, 33]. However, the addition of the AlN inter-layer at the AlGaN/GaN interface causes difficulties in obtaining good ohmic contacts. In order to achieve higher transistor frequencies it is necessary to further increase 2DEG mobility parameter and optimize device design. Therefore, understanding of the factors affecting the $2 \mathrm{DEG}$ properties is very important.

\subsection{Spectroscopic ellipsometry}

Spectroscopic ellipsometry (SE) measures the frequency dependent change of the polarization state of electromagnetic waves upon interaction with a sample. SE provides quantitative information about the complex dielectric function (DF) of layered sample constituents and their thicknesses. SE data is intensity normalized which makes it less sensitive to imperfections of measurements, such as background radiation and power fluctuations of the source.

\subsubsection{Standard ellipsometry}

Standard ellipsometry is commonly used for isotropic samples when no conversion among s-polarized (electric field perpendicular to the plane of incidence) and p-polarized (electric field in the plane of incidence) modes appears. The electric fields of the incoming s- and p-polarized electromagnetic waves $E_{s, p}^{i n}$ and the respective electric fields after in- 
teraction with a sample $E_{s, p}^{\text {out }}$ are connected through the complex reflection (transmission) coefficients $r_{s}$ and $r_{p}\left(t_{s}\right.$ and $\left.t_{p}\right)$, that account for the change in phase and amplitude of the electric fields upon reflection (transmission) from (through) sample

$$
\begin{array}{ll}
E_{s}^{\text {out }}=r_{s} E_{s}^{i n} & \left(E_{s}^{\text {out }}=t_{s} E_{s}^{i n}\right) \\
E_{p}^{\text {out }}=r_{p} E_{p}^{\text {in }} & \left(E_{p}^{\text {out }}=t_{p} E_{p}^{\text {in }}\right) .
\end{array}
$$

Standard ellipsometry data is then expressed as a ratio between the complex reflection (transmission) coefficients

$$
\frac{r_{p}}{r_{s}}=\tan \left(\Psi_{r}\right) \exp \left(i \Delta_{r}\right) \quad\left(\frac{t_{p}}{t_{s}}=\tan \left(\Psi_{t}\right) \exp \left(i \Delta_{t}\right)\right)
$$

In case of bulk isotropic and nontransparent sample, the complex reflection coefficients $r_{s}$ and $r_{p}$ only depend on the DF of the material $\varepsilon$ and the ellipsometry parameters $\Psi_{r}$ and $\Delta_{r}$ can be derived using the Fresnel equations, describing reflection (transmission) coefficients $r_{s}^{*}, r_{p}^{*}\left(t_{s}^{*}, t_{p}^{*}\right)$ for a single interface between two media. The Fresnel equations for reflection (transmission) for the interface between air $\left(\varepsilon_{\text {air }}=1\right)$ and material with DF $\varepsilon$ are

$$
\begin{aligned}
& r_{s}^{*}=\frac{\cos \left(\theta_{i}\right)-\sqrt{\varepsilon} \cos \left(\theta_{m}\right)}{\cos \left(\theta_{i}\right)+\sqrt{\varepsilon} \cos \left(\theta_{m}\right)} \quad\left(t_{s}^{*}=\frac{2 \cos \left(\theta_{i}\right)}{\cos \left(\theta_{i}\right)+\sqrt{\varepsilon} \cos \left(\theta_{m}\right)}\right), \\
& r_{p}^{*}=\frac{\sqrt{\varepsilon} \cos \left(\theta_{i}\right)-\cos \left(\theta_{m}\right)}{\cos \left(\theta_{m}\right)+\sqrt{\varepsilon} \cos \left(\theta_{i}\right)} \quad\left(t_{p}^{*}=\frac{2 \cos \left(\theta_{i}\right)}{\cos \left(\theta_{m}\right)+\sqrt{\varepsilon} \cos \left(\theta_{i}\right)}\right),
\end{aligned}
$$

where $\theta_{i}$ and $\theta_{m}$ are angles between the incoming and the refracted beams, and the surface normal, respectively. For bulk isotropic and nontransparent sample, the complex reflection coefficients $r_{s}, r_{p}$ are equal to Fresnel reflection coefficients $r_{s}^{*}, r_{p}^{*}$, thus

$$
\tan \left(\Psi_{r}\right) \exp \left(i \Delta_{r}\right)=\frac{r_{p}}{r_{s}}=\frac{r_{p}^{*}}{r_{s}^{*}}
$$

When the substrate is transparent and backside reflections occur, or in case of transparent multilayered samples, the parameters $\Psi_{r}$ and $\Delta_{r}$ cannot be simply derived from the Fresnel equations (Eqn. 1.4) and the DFs together with thicknesses of all sample constituents have to be taken into account. 


\subsubsection{Generalized ellipsometry}

For samples with an in-plane optical anisotropy, conversion of s-polarized light into ppolarized and vice versa occurs upon reflection (transmission). In such case, the relations between the outcoming and incoming electric fields become

$$
\begin{gathered}
E_{s}^{\text {out }}=r_{s s} E_{s}^{\text {in }}+r_{p s} E_{p}^{\text {in }} \quad\left(E_{s}^{\text {out }}=t_{s s} E_{s}^{\text {in }}+t_{p s} E_{p}^{\text {in }}\right), \\
E_{p}^{\text {out }}=r_{s p} E_{s}^{\text {in }}+r_{p p} E_{p}^{\text {in }} \quad\left(E_{p}^{\text {out }}=t_{s p} E_{s}^{\text {in }}+t_{p p} E_{p}^{\text {in }}\right),
\end{gathered}
$$

where the four complex reflection (transmission) coefficients $r_{s s}, r_{p s}, r_{s p}, r_{p p}\left(t_{s s}, t_{p s}, t_{s p}, t_{p p}\right)$ describe the interaction with the sample. In such a case, the two parameters $\Psi_{r}$ and $\Delta_{r}$ ( $\Psi_{t}$ and $\Delta_{t}$ ) cannot fully describe the optical response of the sample and the standard ellipsometry is not sufficient.

Generalized ellipsometry (GE) must be employed for anisotropic samples in order to fully describe their optical response. The GE approach applied in the thesis uses the Mueller matrix (MM) formalism. It employs Stoked vectors, where the polarization state is described by four real valued Stokes parameters

$$
\left(\begin{array}{c}
S_{1} \\
S_{2} \\
S_{3} \\
S_{4}
\end{array}\right)=\left(\begin{array}{c}
I_{p}+I_{S} \\
I_{p}-I_{s} \\
I_{+45}-I_{-45} \\
I_{\sigma+}-I_{\sigma-}
\end{array}\right),
$$

where $I_{s}, I_{p}$ are the intensities of the s- and p-polarized modes, $I_{+45}, I_{-45}$ are the intensities of the $+45^{\circ}$ and the $-45^{\circ}$ rotated (with respect to the plane of incidence) linearly polarized light modes, and $I_{\sigma+}, I_{\sigma-}$ are the intensities of left-hand and right-hand circularly polarized light modes. MM ellipsometry measures the $4 \times 4$ transformation matrix $\mathbf{M}$ which relates the Stokes vector of outcoming beam $\overrightarrow{\mathrm{S}}^{\text {out }}$ with the Stokes vector of incoming beam $\overrightarrow{\mathbf{S}}^{\text {in }}$

$$
\left(\begin{array}{l}
S_{1}^{\text {out }} \\
S_{2}^{\text {out }} \\
S_{3}^{\text {out }} \\
S_{4}^{\text {out }}
\end{array}\right)=\left(\begin{array}{llll}
M_{11} & M_{12} & M_{13} & M_{14} \\
M_{21} & M_{22} & M_{23} & M_{24} \\
M_{31} & M_{32} & M_{33} & M_{34} \\
M_{41} & M_{42} & M_{43} & M_{44}
\end{array}\right)\left(\begin{array}{l}
S_{1}^{\text {in }} \\
S_{2}^{\text {in }} \\
S_{3}^{\text {in }} \\
S_{4}^{\text {in }}
\end{array}\right) .
$$

Since the Stokes vectors contain real-valued parameters, the MM elements $\left(M_{i, j}\right.$, where $\left.i, j=1,2,3,4\right)$ are also real numbers. The MM is commonly normalized by the $\mathrm{M}_{11}$ element, which carries the information about the total reflection/transmission 
of a sample. For in-plane isotropic samples measurements of MM provide redundant information and standard ellipsometry is sufficient.

\subsubsection{Dielectric function in the infrared and terahertz spectral ranges}

Charged particles subjected to an electric and magnetic fields varying in time are displaced from their equilibrium positions which results in a polarization of the material. For most crystalline materials the magnetic field can be neglected (magnetic permeability is 1 ) and it is enough to consider only the effect of the electric field. Assuming a linear material response the external electric field $\overrightarrow{\mathbf{E}}$ is related to the displacement field $\overrightarrow{\mathbf{D}}$ through the material polarization $\overrightarrow{\mathbf{P}}$ as

$$
\overrightarrow{\mathbf{D}}=\varepsilon_{0} \overrightarrow{\mathbf{E}}+\overrightarrow{\mathbf{P}}=\varepsilon_{0}(\mathbf{I}+\chi) \overrightarrow{\mathbf{E}}=\varepsilon_{0} \varepsilon \overrightarrow{\mathbf{E}},
$$

where $\mathbf{I}$ is the unit matrix and the polarization of the material depends on the electric field $\overrightarrow{\mathbf{E}}$ through the relation

$$
\overrightarrow{\mathbf{P}}=\varepsilon_{0} \chi \overrightarrow{\mathbf{E}}
$$

and $\chi$ is an electric susceptibility tensor. The external electric field $\overrightarrow{\mathbf{E}}$ is transformed into the displacement field in the material $\overrightarrow{\mathbf{D}}$ by the dielectric function (DF) $\varepsilon$, which in the general case takes the form of a second rank tensor with non-vanishing off-diagonal elements

$$
\varepsilon=\mathbf{I}+\chi=\left(\begin{array}{ccc}
\varepsilon_{x x} & \varepsilon_{x y} & \varepsilon_{x z} \\
\varepsilon_{y x} & \varepsilon_{y y} & \varepsilon_{y z} \\
\varepsilon_{z x} & \varepsilon_{z y} & \varepsilon_{z z}
\end{array}\right) .
$$

The total polarization of the material is generally a superposition of all possible contributions, thus the DF tensor can be written as

$$
\varepsilon=\mathbf{I}+\sum_{i} \chi_{i}
$$

When a bound particle with charge $q$ and mass tensor $\mathbf{m}$ is exposed to an alternating electric field $\overrightarrow{\mathbf{E}}$, its movement can be derived from the classical equation of motion

$$
\mathbf{m} \frac{d^{2} \overrightarrow{\mathbf{r}}}{d t^{2}}=q \overrightarrow{\mathbf{E}}-\mathbf{m} \gamma \frac{d \overrightarrow{\mathbf{r}}}{d t}-\mathbf{m} \omega_{0}^{2} \overrightarrow{\mathbf{r}}
$$

where $\overrightarrow{\mathbf{r}}$ is the position vector, $\gamma=\tau^{-1}$ is the broadening tensor which is inverse of the scattering time tensor $\tau, \omega_{0}$ is the resonant frequency tensor. Using a time-harmonic electric field $\overrightarrow{\mathbf{E}}=\left(E_{x}, E_{y}, E_{z}\right) e^{i \omega t}$ and assuming a time harmonic response of the charged particles $\overrightarrow{\mathbf{r}}=(x, y, z) e^{i \omega t}$, Eqn. 1.13 describes a well-known harmonic Lorentz oscilla- 
tor. Plugging in the solution of Eqn. 1.13 into the expression for the polarization vector $\overrightarrow{\mathbf{P}}=q n \overrightarrow{\mathbf{r}}$ ( $n$ - number of oscillators per unit volume), and using Eqn. 1.10, the electric susceptibility tensor can be derived

$$
\chi=\frac{n q^{2} \mathbf{m}^{-1}}{\varepsilon_{0}}\left(\omega_{0}^{2}-\omega^{2} \mathbf{I}-i \omega \gamma\right)^{-1}
$$

\section{Phonon contribution}

The dielectric response of polar crystalline materials in the IR spectral range is governed by the contribution from IR-active polar phonon modes. For materials with a single IRactive phonon mode or materials that possess several phonon modes that are not coupled, the electric susceptibilities $\chi^{L}$ are well described by the harmonic Lorentz oscillator model (Eqn. 1.14), which are often expressed in the form

$$
\chi^{L}=\sum_{i} A_{i}\left(\omega_{0, i}^{2}-\omega^{2} \mathbf{I}-i \omega \gamma_{i}\right)^{-1}
$$

where the sum runs over all active phonon modes and $A_{i}$ represents the oscillator strength of $i$-th phonon. When anharmonic coupling between phonon modes is present, the dielectric response cannot be well-described as a simple sum of harmonic oscillators with independent broadening parameters (Eqn. 1.15). It was shown that for polar multi-phonon materials, such as sapphire or III group nitrides, a factorized four-parameter semi-quantum model, for the dielectric response, provides a much better match to the experimentally determined results $[34,35]$. It allows for different broadenings of a LO and a TO phonon modes. For uniaxial polar semiconductors, such as wurtzite structure III-group nitrides, the factorized model DF tensor in the Cartesian coordinates has the following form

$$
\begin{gathered}
\varepsilon^{L}=\mathbf{I}-\chi^{L}=\left(\begin{array}{ccc}
\varepsilon_{\perp}^{L} & 0 & 0 \\
0 & \varepsilon_{\perp}^{L} & 0 \\
0 & 0 & \varepsilon_{\|}^{L}
\end{array}\right), \\
\varepsilon_{j}^{L}=\prod_{i} \frac{\omega_{\mathrm{LO}, i, j}^{2}-\omega^{2}-\mathrm{i} \omega \gamma_{\mathrm{LO}, i, j}}{\omega_{\mathrm{TO}, i, j}^{2}-\omega^{2}-\mathrm{i} \omega \gamma_{\mathrm{TO}, i, j}} \quad(j=\perp, \|),
\end{gathered}
$$

where $\perp$ and $\|$ stands for polarization perpendicular and parallel to the optical axis, respectively. The factorized DF tensor elements contain LO and TO phonon frequencies denoted as $\omega_{L O}$ and $\omega_{L O}$, respectively, and corresponding broadening parameters as $\gamma_{L O}$ and $\gamma_{T O}$, respectively. Note that the DF tensor, calculated from the electric susceptibility, derived using Eqn. 1.15, is a partial fraction decomposition of the factorized DF tensor (Eqn. 1.16), when the broadening parameters of the LO and TO modes are equal 
$\left(\gamma_{L O}=\gamma_{T O}\right)$. To keep a physical meaning of the factorized model DF tensor (Eqn. 1.16), i.e. $\Im \mathrm{m}\left[\varepsilon_{i}\right] \geq 0$, the first generalized Lowndes's condition must be satisfied [36]

$$
\sum_{i}\left(\gamma_{L O, i}-\gamma_{T O, i}\right) \geq 0
$$

\section{Free charge carrier contributions (non magnetic case)}

Contribution to the DF tensor from unbound charged particles, such as FCCs, can be easily obtained from the DF tensor, derived from the classical equation of motion (Eqn. 1.14), by assuming that there is no restoring force that implies $\omega_{0} \equiv 0$. The electric susceptibility tensor then reads

$$
\chi^{F C C}=-\sum_{i} \frac{n_{i} q_{i}^{2} \mathbf{m}_{\mathbf{i}}^{-1}}{\varepsilon_{0}}\left(\omega^{2} \mathbf{I}+i \omega \gamma_{i}\right)^{-1},
$$

which resembles the well-known Drude model. When modeling FCC contributions in semiconductor materials, the sum can run over two constituents, holes (p-type conductivity) and electrons (n-type conductivity). The broadening tensor $\gamma_{i}$ can be shown to depend on FCCs mobility $\boldsymbol{\mu}_{i}$ and effective mass $\mathbf{m}_{\mathbf{i}}$ tensors

$$
\gamma_{i}=q\left(\mathbf{m}_{\mathrm{i}} \mu_{i}\right)^{-1}
$$

When material possesses only one type of conductivity, the electric susceptibility tensor for FCCs (Eqn. 1.18) can be written as

$$
\chi^{F C C}=-\omega_{p}^{2}\left(\omega^{2} \mathbf{I}+i \omega \gamma\right)^{-1}
$$

It has two independent parameters, the plasma broadening tensor $\gamma$ (Eqn. 1.19) and the plasma frequency tensor defined as

$$
\omega_{p}^{2}=n q^{2} \mathbf{m}^{-1} / \varepsilon_{0}
$$

\section{High frequency contributions}

Inter-band electronic excitations at higher frequencies also contribute to the dielectric response, and can be observed in the IR spectral range as an offset in the DF tensor elements. The high frequency contribution is included by adding a frequency independent parameter $\varepsilon_{\infty}$, which in general is also a tensor quantity. Then the DF tensor including 
the phonon and the FCC contributions for uniaxial polar crystal can be written as

$$
\varepsilon_{j}=\varepsilon_{\infty, j}+\chi_{j}^{L}+\chi_{j}^{F C C}=\varepsilon_{\infty, j}\left(\prod_{i} \frac{\omega_{\mathrm{LO}, i, j}^{2}-\omega^{2}-\mathrm{i} \omega \gamma_{\mathrm{LO}, i, j}}{\omega_{\mathrm{TO}, i, j}^{2}-\omega^{2}-\mathrm{i} \omega \gamma_{\mathrm{TO}, i, j}}-\frac{\omega_{p, j}^{*}{ }^{2}}{\left(\omega^{2}+i \omega \gamma_{j}\right)}\right),
$$

where $j=\perp, \|$ and $\omega_{p, j}^{*}=\sqrt{n q^{2} /\left(\varepsilon_{0, j} \varepsilon_{\infty, j} m_{j}\right)}$ is the screened plasma frequency. Eqn. 1.22 assumes that the optical axes of the phonon and the FCC electric susceptibilities are collinear.

\subsubsection{Optical Hall effect}

The OHE describes the magnetic field-induced optical birefringence of conductive materials, at IR and THz frequencies, due to the motion of FCCs under the influence of the Lorentz force. It appears when an external magnetic field augments off-diagonal elements in the DF tensors of the conductive materials within sample, which results in conversion between s- and p-polarized modes upon reflection (transmission). The electrical Hall effect could be considered as a low frequency case $(\omega \rightarrow 0)$ of the OHE.

The external magnetic field has a negligible effect on the optical phonons since lattice ions have much higher masses compared with electrons and holes. At IR and THz spectral ranges the DF tensor of conductive materials, when exposed to an external magnetic field, can be written as a sum of three terms: (i) the magnetic field independent high frequency tensor $\varepsilon_{\infty}$, (ii) lattice electric susceptibility tensor $\chi^{L}$ and (iii) a magneto-optic FCC electric susceptibility tensor $\chi^{F C C-M O}$,

$$
\varepsilon=\varepsilon_{\infty}+\chi^{L}+\chi^{F C C-M O}
$$

The magneto-optic FCC contribution $\chi^{F C C-M O}$ can be derived from the classical equation of motion (Eqn. 1.13) for unbound charged particles $\left(\omega_{0} \equiv 0\right)$ with an additional term for the Lorentz force contribution

$$
\mathbf{m} \frac{d^{2} \overrightarrow{\mathbf{r}}}{d t^{2}}=q \overrightarrow{\mathbf{E}}-\mathbf{m} \gamma \frac{d \overrightarrow{\mathbf{r}}}{d t}-q\left(\frac{d \overrightarrow{\mathbf{r}}}{d t} \times \mathbf{B}\right) .
$$

Using the solution of Eqn. 1.24 the magneto-optic electric susceptibility tensor can be derived

$$
\chi^{F C C-M O}=-\omega_{p}^{2}\left(\omega^{2} \mathbf{I}+i \omega \gamma_{p}-i \omega \omega_{c}\left(\begin{array}{ccc}
0 & -b_{z} & b_{y} \\
b_{z} & 0 & -b_{x} \\
-b_{y} & b_{x} & 0
\end{array}\right)\right)^{-1}
$$


where the magnetic field vector is defined as $\overrightarrow{\mathbf{B}}=|\overrightarrow{\mathbf{B}}|\left(b_{x}, b_{y}, b_{z}\right)$ and $\boldsymbol{\omega}_{c}=q|\overrightarrow{\mathbf{B}}| \mathbf{m}^{-1}$ is a cyclotron frequency tensor. The FCC response under the influence of the magnetic field is non-time-reciprocal, which results in an antisymmetric magneto-optic FCC electric susceptibility tensor $\chi^{F C C-M O}$ (Eqn. 1.25), and as a result an antisymmetric DF tensor $\varepsilon$ (Eqn. 1.23). For isotropic materials, the magneto-optic FCC electric susceptibility $\chi^{F C C-M O}$ contains three independent parameters: the plasma frequency $\omega_{p}$, the plasma broadening $\gamma_{p}$ and the cyclotron frequency $\omega_{c}$, which themselves depend on the FCC concentration $\mathrm{N}$, mobility $\mu$ and effective mass $m$ parameters (Eqns. 1.19, 1.21).

\subsubsection{Cavity-enhanced optical Hall effect}

The magnitude of the conversion among s- and p-polarized modes due to the OHE in the $\mathrm{THz}$ spectral range strongly depends on the FCC sheet density and mobility parameters. For samples with low FCC mobilities and sheet densities, the OHE contribution to the SE spectra is negligible and the sensitivity to the FCC properties in OHE measurement is limited. It has been shown that the use of a backside cavity with a highly reflective backside surface can enhance the OHE in HEMT structures and EG, as a result of the formation of Fabri-Pérot modes, within the sample-cavity system [37-39]. An optical scheme of the cavity-enhanced (CE)-OHE measurement for a sample containing a transparent substrate and a conductive layer on top is shown in Figure 1.3.

At those frequencies where the minima of the reflection occur, a strong conversion among s- and p-polarized modes take place as a result of the retro-reflections in combination with the OHE within the sample-cavity optical system. Furthermore, control of the gap thickness parameter provides an additional degree of freedom as an input for the CE-OHE measurements. Employment of the cavity can dispense the need of high magnetic fields and even common permanent magnets can provide magnetic field strengths sufficient to cause detectable OHE and as a result sensitivity to the FCC parameters.

\subsubsection{Ellipsometric data analysis}

Physical parameters of interest are extracted by fitting optical model based on parametrized DF tensors to SE data. The three main steps of the data analysis procedure are the definition of the DF tensors, construction of the optical model and fitting of a optical model to the SE data.

DF tensors of sample constituents can be provided as tabulated sets of parameters, parametrized empirical tensors and parametrized physical model based tensors. For materials which have well-established optical properties DF tensors are usually taken from literature or databases. 


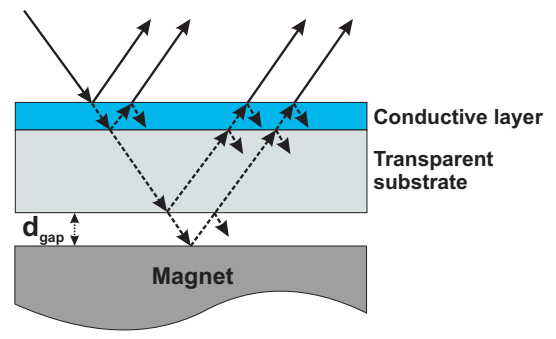

Figure 1.3: Optical scheme of a cavity-enhanced optical Hall effect measurement.

The optical model assumes a set of layers with perfectly planar interfaces, constant thicknesses and optical properties described by DF tensors. The simplest case for analysis of SE spectra is when the sample is a non-transparent isotropic bulk substrate. In such case, standard ellipsometry is sufficient to account for the optical response of the sample. $\Psi_{r}$ and $\Delta_{r}$ spectra can be derived from the complex Fresnel reflection coefficients for sand p-polarized light $\left(r_{s}^{*}, r_{p}^{*}\right.$ in Eqn. 1.4), which directly depend on the DF of the substrate material

$$
\rho=\tan \left(\Psi_{r}\right) \exp \left(i \Delta_{r}\right)=\frac{r_{p}^{*}}{r_{s}^{*}}=\left(\frac{\sqrt{\varepsilon} \cos \left(\theta_{i}\right)-\cos \left(\theta_{m}\right)}{\sqrt{\varepsilon} \cos \left(\theta_{i}\right)+\cos \left(\theta_{m}\right)}\right) /\left(\frac{\cos \left(\theta_{i}\right)-\sqrt{\varepsilon} \cos \left(\theta_{m}\right)}{\cos \left(\theta_{i}\right)+\sqrt{\varepsilon} \cos \left(\theta_{m}\right)}\right) .
$$

The inverse procedure can be applied to calculate the DF of the substrate materials directly from the SE data. In such instances, the so-called pseudo-DF $\langle\varepsilon\rangle$ is obtained

$$
<\varepsilon>=\sin ^{2}\left(\theta_{i}\right)\left[1+\tan ^{2}\left(\theta_{i}\right)\left(\frac{1-\rho}{1+\rho}\right)^{2}\right],
$$

which is identical to the DF $\varepsilon$ in the case of a non-transparent isotropic bulk sample. A lineshape analysis of the pseudo-DF based on physical models provides information on the physical properties of the substrate material.

When sample consists of a set of transparent layers the ellipsometric data depends on the DFs of all sample constituents and their thicknesses. In such case, the pseudo-DF represents a convolution of the dielectric properties and the thicknesses of all sample constituents. Calculation of the sample's optical response has to account for the multireflections within the sample structure and the use of calculations based on the complex Fresnel reflection coefficients become inconvenient. Furthermore, if the substrate or any of the layers is anisotropic and therefore has to be characterized by a DF tensor rather than a DF the use of Fresnel equations is not possible. 
The $4 \times 4$ partial transfer matrices derived from Berreman relation provides a very powerful method for modeling the optical response of anisotropic layered structures [40, 41]. The transfer matrix $\mathbf{T}_{\mathbf{p}}$ connects the tangential electric $E_{x}, E_{y}$ and magnetic $H_{x}, H_{y}$ fields of two planar interfaces, separated by the distance $d$, within a medium described by the DF tensor $\varepsilon$ (Eqn. 1.11) (see Figure 1.4)

$$
\begin{gathered}
\left(\begin{array}{c}
E_{x} \\
E_{y} \\
H_{x} \\
H_{y}
\end{array}\right)_{z=d}=\mathbf{T}_{\mathbf{p}}\left(\begin{array}{c}
E_{x} \\
E_{y} \\
H_{x} \\
H_{y}
\end{array}\right)_{z=0}, \\
\mathbf{T}_{\mathbf{p}}=\exp \left(i \frac{\omega}{c} \boldsymbol{\Delta}_{B} d\right), \\
\boldsymbol{\Delta}_{\boldsymbol{B}}=\left(\begin{array}{cccc}
-K_{x x} \frac{\varepsilon_{z x}}{\varepsilon_{z z}} & -K_{x x} \frac{\varepsilon_{z y}}{\varepsilon_{z z}} & 0 & 1-\frac{K_{x x}^{2}}{\varepsilon_{z z}} \\
0 & 0 & -1 & 0 \\
\varepsilon_{y z} \frac{\varepsilon_{z x}}{\varepsilon_{z z}}-\varepsilon_{y x} & K_{x x}^{2}-\varepsilon_{y y}+\varepsilon_{y z} \frac{\varepsilon_{z y}}{\varepsilon_{z z}} & 0 & K_{x x} \frac{\varepsilon_{x z}}{\varepsilon_{z z}} \\
\varepsilon_{x x}-\varepsilon_{x z} \frac{\varepsilon_{z x}}{\varepsilon_{z z}} & \varepsilon_{x y}-\varepsilon_{x z} \frac{\varepsilon_{z z}}{\varepsilon_{z z}} & 0 & -K_{x x} \frac{\varepsilon_{x z}}{\varepsilon_{z z}}
\end{array}\right),
\end{gathered}
$$

with $K_{x x}=\frac{\omega}{c} n_{i} \sin \left(\theta_{i}\right)$, where $c$ is the speed of light in free space and $n_{i}$ is the refractive index of an incident medium (commonly air, thus $n_{i}=1$ ). Propagation of light in a multilayered sample can be expressed as a product of transfer matrices for all layers. Using the $4 \times 4$ partial transfer matrix method, one can derive the complex reflection $r_{s s}, r_{p s}, r_{s p}, r_{p p}$ and transmission $t_{s s}, t_{p s}, t_{s p}, t_{p p}$ coefficients of the modeled sample, which are used to calculate the SE parameters.

Fitting of parameterized optical models to SE data is performed using a linear regression method. Optical model parameters are varied to get the best-match between optical model and corresponding SE data sets. The mismatch between calculated and SE data sets, weighted by the experimental errors, is expressed by the mean square error (MSE) parameters

$$
\begin{gathered}
M S E^{\Psi, \Delta}=\sqrt{\frac{1}{2 S-K} \sum_{i=1}^{S}\left(\frac{\Psi_{i}^{E}-\Psi_{i}^{G}}{\sigma_{\Psi_{i}^{E}}}\right)^{2}+\left(\frac{\Delta_{i}^{E}-\Delta_{i}^{G}}{\sigma_{\Delta_{i}^{E}}}\right)^{2}}, \\
M S E^{M M}=\sqrt{\frac{1}{a b S-K} \sum_{i=1}^{a} \sum_{j=1}^{b} \sum_{k=1}^{S}\left(\frac{M_{i, j, k}^{E}-M_{i, j, k}^{G}}{\sigma_{M_{i, j, k}^{E}}^{E}}\right)^{2}},
\end{gathered}
$$

where $M S E^{\Psi, \Delta}$ is used in case of standard ellipsometry and $M S E^{\Psi, \Delta}$ for MM ellipsometry, 


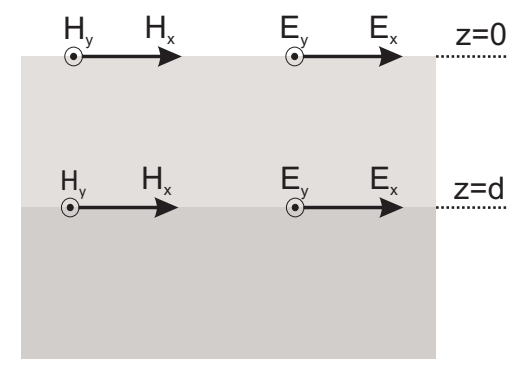

Figure 1.4: Propagation of tangential electric $E_{x}, E_{y}$ and magnetic $H_{x}, H_{y}$ fields employed in the $4 \times 4$ partial transfer matrix formalism.

$S$ and $K$ are the total number of spectral points and fitting parameters in the optical model, respectively, $\sigma_{\Psi_{i}^{E}}, \sigma_{\Delta_{i}^{E}}, \sigma_{M_{i, j, k}}$ are experimental standard deviations of the $\Psi_{i}^{E}, \Delta_{i}^{E}$ and $M_{i, j, k}$ data points, respectively, the indices $a, b$ indicate the total number rows and columns of the experimental MM. An iteration procedure is applied to minimize the MSE by varying optical model parameters. The best-match model is then used to extract the physical parameters of interest.

\subsubsection{Mid-infrared spectroscopic ellipsometry and optical Hall effect measurements}

MIR SE was introduced in the 90's and has nowadays become a well-established technique capable of performing standard ellipsometry and GE measurements. It was shown that MIR ellipsometry is a powerful method for exploring lattice vibrations and FCC properties in crystalline materials [36].

MIR SE ellipsometry measurements, presented in this thesis, were performed using a commercial Fourier transform IR spectrometer based ellipsometer from the J.A. Woolam Company, operating at a spectral range of $200-7800 \mathrm{~cm}^{-1}$ with a resolution up to $1 \mathrm{~cm}^{-1}$. It is capable of measuring an upper left $4 \times 3$ block of $\mathrm{MM}\left(M_{i j}\right.$, where $i=1,2,3,4$ and $j=1,2,3)$ when operating in GE mode.

MIR OHE measurements, presented in this thesis, were performed on a custom-built MIR ellipsometer equipped with a Fourier transform IR spectrometer $\left(580-7000 \mathrm{~cm}^{-1}\right)$. A technical drawing of the MIR ellipsometer is depicted in Figure 1.5. It operates in the polarizer-sample-rotating analyzer arrangement that allows to perform GE measurements and assesses the upper left $3 \times 3$ block of $\mathrm{MM}\left(M_{i j}\right.$, where $\left.i, j=1,2,3\right)$. The system is equipped with three different detectors: a solid state mercury cadmium telluride photodetector (MCT), a deuterated triglycine sulfate detector (DTGS) and Si bolometer detector. OHE 


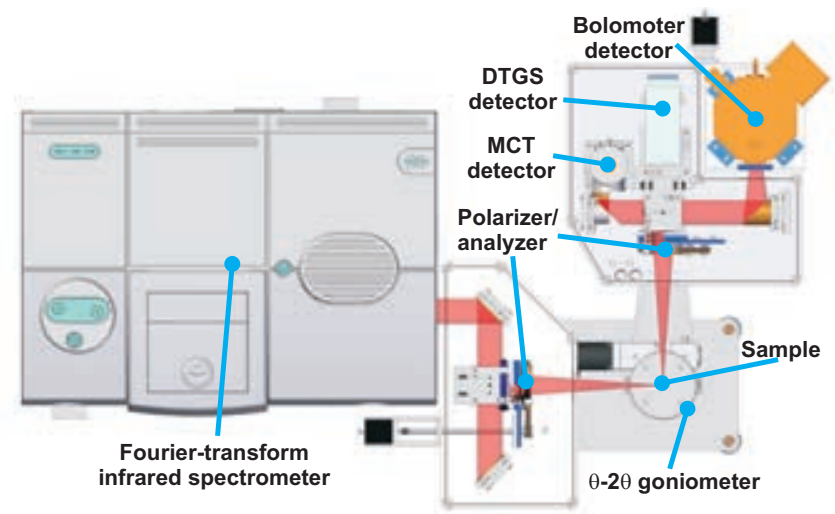

Figure 1.5: Mid-infrared ellipsometer at the University of Nebraska-Lincoln.

measurements were performed using a superconducting closed-cycle magneto-cryostat capable of providing magnetic fields up to $8 \mathrm{~T}$ and sample temperatures from $1.4 \mathrm{~K}$ to room temperature. The MIR ellipsometer is part of the integrated MIR, FIR and THz OHE instrument at the University of Nebraska-Lincoln [42].

\subsubsection{Terahertz optical Hall effect measurements}

The development of $\mathrm{SE}$ at the $\mathrm{THz}$ spectral region has been more challenging than at shorter wavelengths. The main difficulties for the implementation comes from a lack of high intensity and quality sources, a high background black-body radiation contamination and limited choices for polarizing optical elements $[43,44]$. Since the wavelength of the $\mathrm{THz}$ radiation is in the range of milimeters, optical elements and samples commonly used in SE measurements can cause diffraction [44]. Furthermore, for highly coherent light sources, standing waves can form in the optical system, which has highly detrimental effect on the performance of the ellipsometer. However, despite the challenges, $\mathrm{THz}$ ellipsometry is a rapidly developing field due to its potential in application as a characterization tool for novel solid state materials and new high frequency electronic devices. The sensitivity to the optical properties of materials at $\mathrm{THz}$ frequencies opens a broad field of applications to study different physical phenomena, such as spin-transitions, collective modes in biological molecules and local-FCC oscillations [43].

OHE measurements in the $\mathrm{THz}$ range, presented in this thesis, were performed using a custom-built frequency-domain THz ellipsometer at the Terahertz Materials Analysis Center in Linköping University. A technical drawing of the system is depicted in Figure 1.6. The THz ellipsometer employs a frequency tunable, continuous wave backward- 


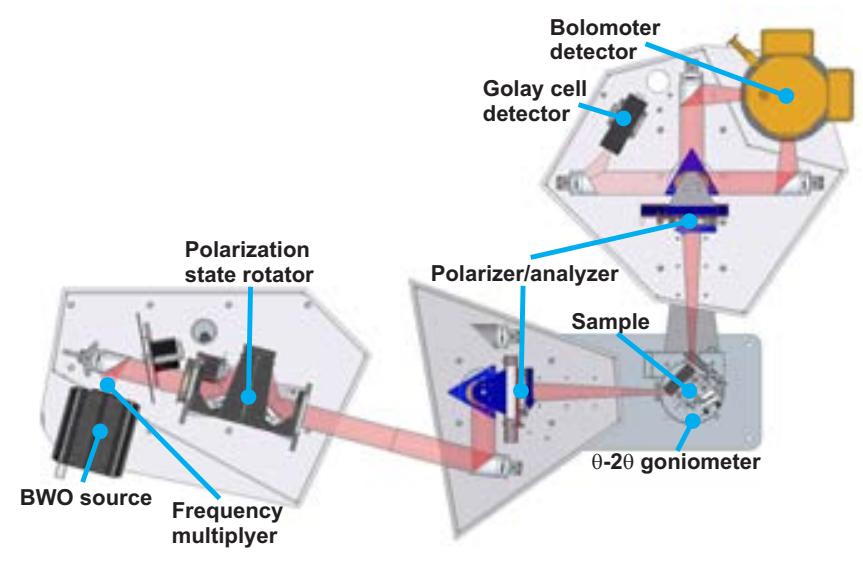

Figure 1.6: Terahertz ellipsometer at the Terahertz Materials Analysis Center in the Linköping University.

wave oscillator (BWO) source, with output frequencies ranging from $97 \mathrm{GHz}$ to $177 \mathrm{GHz}$, (bandwidth $\sim 1 \mathrm{MHz}$ ) and output power reaching up to $26 \mathrm{~mW}$. The BWO source can be augmented with Schottky-diode frequency multipliers $(\times 2, \times 3, \times 6)$ which extend the accessible spectral range up to $1000 \mathrm{GHz}(\times 2$ multiplier: $200-350 \mathrm{GHz} ; \times 3$ multiplier: $300-525 \mathrm{GHz} ; \times 6$ multiplier: $650-1000 \mathrm{GHz}$ ). The sample is mounted on a high precision $\theta-2 \theta$ two stage goniometer, which allows to control the angle on incidence from $30^{\circ}$ to $90^{\circ}$. The system contains a Golay cell detector and two liquid helium cooled bolometer detectors. The ellipsometer is equipped with high quality free standing wire grid polarizers and is designed to suppress standing waves by avoiding the presence of parallel reflecting surfaces and using absorbing/scattering surface coverage. The $\mathrm{THz}$ ellipsometer operates in the polarizer-sample-rotating analyzer arrangement, capable of measuring upper-left $3 \times 3$ block of the $\mathrm{MM}\left(M_{i j}\right.$, where $\left.i, j=1,2,3\right)$. 


\section{References}

[1] M. Schubert, T. Hofmann, and C. M. Herzinger, J. Opt. Soc. Am. A 20, 347 (2003).

[2] M. Schubert, P. Kühne, V. Darakchieva, and T. Hofmann, J. Opt. Soc. Am. A 33, 1553 (2016).

[3] C. Riedl, C. Coletti, and U. Starke, J. Phys. D: Appl. Phys. 43, 374009 (2010).

[4] L. I. Johansson and C. Virojanadara, J. Mater. Res. 29, 426 (2014).

[5] C. Bouhafs, V. Darakchieva, I. Persson, A. Tiberj, P. A. Persson, M. Paillet, A. A. Zahab, P. Landois, S. Juillaguet, S. Schöche, and M. Schubert, J. Appl. Phys. 117, o85701 (2015).

[6] T. Yager, A. Lartsev, K. Cedergren, R. Yakimova, V. Panchal, O. Kazakova, A. Tzalenchuk, K. H. Kim, Y. W. Park, S. Lara-Avila, and S. Kubatkin, AIP Adv. 5, o87134 (2015).

[7] M. Beshkova, L. Hultman, and R. Yakimova, Vacuum 128, 186 (2016).

[8] Y. M. Lin, C. Dimitrakopoulos, K. A. Jenkins, D. B. Farmer, H. Y. Chiu, A. Grill, and P. Avouris, Science 327, 662 (2010).

[9] Y. M. Lin, A. Valdes-Garcia, S. J. Han, D. B. Farmer, I. Meric, Y. Sun, Y. Wu, C. Dimitrakopoulos, A. Grill, P. Avouris, and K. A. Jenkins, Science 332, 1294 (2011).

[10] S. Mammadov, J. Ristein, R. J. Koch, M. Ostler, C. Raidel, M. Wanke, R. Vasiliauskas, R. Yakimova, and T. Seyller, 2D Materials 1, 035003 (2014).

[11] S. Knight, T. Hofmann, C. Bouhafs, N. Armakavicius, P. Kühne, V. Stanishev, I. G. Ivanov, R. Yakimova, S. Wimer, M. Schubert, and V. Darakchieva, Sci. Rep. 7 (2017). 
[12] C. Riedl, C. Coletti, T. Iwasaki, A. Zakharov, and U. Starke, Phys. Rev. Lett. 103, 246804 (2009).

[13] S. Tanabe, Y. Sekine, H. Kageshima, and H. Hibino, Jpn. J. Appl. Phys. 51, o2BNo2 (2012).

[14] S. Watcharinyanon, C. Virojanadara, J. Osiecki, A. Zakharov, R. Yakimova, R. Uhrberg, and L. I. Johansson, Surf. Sci. 605, 1662 (2011).

[15] A. G. Bhuiyan, A. Hashimoto, and A. Yamamoto, J. Appl. Phys. 94, 2779 (2003).

[16] X. Wang, S. Liu, N. Ma, L. Feng, G. Chen, F. Xu, N. Tang, S. Huang, K. J. Chen, S. Zhou, and B. Shen, Appl. Phys. Express 5, 015502 (2012).

[17] S. Schöche, T. Hofmann, V. Darakchieva, N. Ben Sedrine, X. Wang, A. Yoshikawa, and M. Schubert, J. Appl. Phys. 113, 013502 (2013).

[18] A. Kasic, M. Schubert, S. Einfeldt, D. Hommel, and T. Tiwald, Phys. Rev. B 62, 7365 (2000).

[19] C. Arguello, D. L. Rousseau, and S. P. S. Porto, Phys. Rev. 181, 1351 (1969).

[20] T. Hofmann, V. Darakchieva, B. Monemar, H. Lu, W. Schaff, and M. Schubert, J. Electron. Mater. 37, 611 (2008).

[21] A. Kasic, M. Schubert, Y. Saito, Y. Nanishi, and G. Wagner, Phys. Rev. B 65, 115206 (2002).

[22] V. Darakchieva, M. Schubert, J. Birch, A. Kasic, S. Tungasmita, T. Paskova, and B. Monemar, Physica B: Condensed Matter 340, 416 (2003).

[23] S. Schöche, P. Kühne, T. Hofmann, M. Schubert, D. Nilsson, A. KakanakovaGeorgieva, E. Janzén, and V. Darakchieva, Appl. Phys. Lett. 103, 212107 (2013).

[24] J. Wu, J. Appl. Phys. 106, 5 (2009).

[25] H. Xing, S. Keller, Y. Wu, L. McCarthy, I. Smorchkova, D. Buttari, R. Coffie, D. Green, G. Parish, S. Heikman, and L. Shen, J. Phys.: Condens. Matter 13, 7139 (2001).

[26] J. A. del Alamo and J. Joh, Microelectron Reliab. 49, 1200 (2009).

[27] F. Roccaforte, P. Fiorenza, G. Greco, R. L. Nigro, F. Giannazzo, A. Patti, and M. Saggio, Phys. Status Solidi C 211, 2063 (2014). 
[28] F. Roccaforte, P. Fiorenza, G. Greco, M. Vivona, R. L. Nigro, F. Giannazzo, A. Patti, and M. Saggio, Appl. Surf. Sci. 301, 9 (2014).

[29] J. W. Chung, W. E. Hoke, E. M. Chumbes, and T. Palacios, IEEE Electron Device Lett. 31, 195 (2010).

[30] V. Polyakov, V. Cimalla, V. Lebedev, K. Köhler, S. Müller, P. Waltereit, and O. Ambacher, Appl. Phys. Lett. 97, 142112 (2010).

[31] J.-T. Chen, I. Persson, D. Nilsson, C.-W. Hsu, J. Palisaitis, U. Forsberg, P. O. Persson, and E. Janzén, Appl. Phys. Lett. 106, 251601 (2015).

[32] X. Wang, G. Hu, Z. Ma, J. Ran, C. Wang, H. Xiao, J. Tang, J. Li, J. Wang, Y. Zeng, et al., J. Cryst. Growth 298, 835 (2007).

[33] J.-T. Chen, C.-W. Hsu, U. Forsberg, and E. Janzén, J. Appl. Phys. 117, 085301 (2015).

[34] M. Schubert, T. Tiwald, and C. Herzinger, Phys. Rev. B 61, 8187 (2000).

[35] F. Gervais and B. Piriou, J. Phys. C. Solid State Phys. 7, 2374 (1974).

[36] M. Schubert, Infrared Ellipsometry on semiconductor layer structures: Phonons, Plasmons, and Polaritons, 209 (Springer Science \& Business Media, 2004).

[37] S. Knight, S. Schöche, V. Darakchieva, P. Kühne, J.-F. Carlin, N. Grandjean, C. Herzinger, M. Schubert, and T. Hofmann, Opt. Lett. 40, 2688 (2015).

[38] N. Armakavicius, T. Chen Jr, T. Hofmann, S. Knight, P. Kühne, D. Nilsson, U. Forsberg, E. Janzén, and V. Darakchieva, Phys. Status Solidi C 13, 369 (2016).

[39] N. Armakavicius, C. Bouhafs, V. Stanishev, P. Kühne, R. Yakimova, S. Knight, T. Hofmann, M. Schubert, and V. Darakchieva, Appl. Surf. Sci. 421, 357 (2017).

[40] M. Schubert, Phys. Rev. B 53, 4265 (1996).

[41] H. Fujiwara, Principles of Spectroscopic Ellipsometry, (John Wiley \& Sons, Ltd, 2007).

[42] P. Kühne, C. M. Herzinger, M. Schubert, J. A. Woollam, and T. Hofmann, Rev. Sci. Instrum. 85, 071301 (2014).

[43] T. Hofmann, C. Herzinger, J. Tedesco, D. Gaskill, J. Woollam, and M. Schubert, Thin Solid Films 519, 2593 (2011).

[44] M. Neshat and N. P. Armitage, J. Infrared Millim. Terahertz Waves 34, 682 (2013). 



\section{List of abbreviations}

AFM

Al

AlN

AlGaN

BL

CE

DF

EG

FCC

Ga

$\mathrm{GaN}$

GE

$\mathrm{GHz}$

HEMT

InGaN

In

InN

IR

LO

MIR

ML atomic force microscopy

aluminum

aluminum nitride

aluminum gallium nitride

bilayer

cavity-enhanced

dielectric function

epitaxial graphene

free charge carrier

gallium

gallium nitride

generalized ellipsometry

gigahertz

high electron mobility transistor

indium gallium nitride

indium

indium nitride

infrared

longitudinal optical

mid-infrared

monolayer 
MM

mueller matrix

MSE

mean square error

$\mathbf{N}$

nitrogen

OHE

optical Hall effect

SE

spectroscopic ellipsometry

Si

silicon

$\mathrm{SiC}$

silicon carbide

QFS

quasi-free-standing

THz

terahertz

TO

transverse optical

2DEG

two-dimensional electron gas 


\section{Part II}

\subsection{Publications included in the thesis}

\section{Paper I}

Philipp Kühne, Vallery Stanishev, Nerijus Armakavicius, Mathias Schubert, Vanya Darakchieva

"Terahertz frequency-domain ellipsometry"

in manuscript

I was extensively involved in the development of the terahertz ellipsometry instrumentation at the Linköping University. I was involved in designing, assembling and testing of the instrument. I have also performed the optical Hall effect measurements and the data analysis of the AlGaN/GaN HEMT structure presented in the paper.

\section{Paper II}

Nerijus Armakavicius, Chamseddine Bouhafs, Vallery Stanishev, Philipp Kühne, Rositsa Yakimova, Sean Knight, Tino Hofmann, Mathias Schubert, Vanya Darakchieva "Cavity-enhanced optical Hall effect in epitaxial graphene detected at terahertz frequencies" Applied Surface Science, 421, 357-360 (2017)

I took part in the data analysis and discussions of the results. I was also active in writing of the paper. 


\section{Paper III}

Nerijus Armakavicius, Philipp Kühne, Chamseddine Bouhafs, Vallery Stanishev, Sean Knight, Rositsa Yakimova, Alexei Zakharov, Camilla Colleti, Mathias Schubert, Vanya Darakchieva

"Study of anisotropic transport in as-grown and quasi-free-standing epitaxial graphene by terahertz cavity enhanced optical Hall effect"

in manuscript

I have done the optical Hall effect measurements and was extensively involved in the data analysis and interpretation of the results. I also wrote the paper.

\section{Paper IV}

Nerijus Armakavicius, Vallery Stanishev, Sean Knight, Philipp Kühne, Mathias Schubert, Vanya Darakchieva

"Anisotropic electron effective mass and mobility parameters in $\mathrm{In}_{0.33} \mathrm{Ga} a_{0.67} \mathrm{~N}$ determined by midinfrared optical Hall effect"

in manuscript

I took part in mid-infrared ellipsometry and optical Hall effect measurements. I was extensively involved in the data analysis and interpretation of the results. I also wrote the paper.

\section{Paper V}

Nerijus Armakavicius, Jr-Tai Chen, Tino Hofmann, Sean Knight, Philipp Kühne, Daniel Nilsson, Urban Forsberg, Erik Janzén, Vanya Darakchieva

"Properties of two-dimensional electron gas in AlGaN/GaN HEMT structures determined by cavity-enhanced THz optical Hall effect"

Physica Status Solidi C, 13, 369-373 (2016)

I was active in the optical Hall effect measurements, data analysis and interpretation of the results. I also wrote the paper. 


\subsection{Publications not included in the thesis}

Sean Knight, Tino Hofmann, Chamseddine Bouhafs, Nerijus Armakavicius, Philipp Kühne, Vallery Stanishev, Ivan G. Ivanov, Rositsa Yakimova, Shawn Wimer, Mathias Schubert, Vanya Darakchieva

"In-situ terahertz optical Hall effect measurements of ambient effects on free charge carrier properties of epitaxial graphene"

Scientific Reports, 7, (2017) 



\section{Papers}

The papers associated with this thesis have been removed for copyright reasons. For more details about these see:

http://urn.kb.se/resolve?urn=urn:nbn:se:liu:diva-142220 